\title{
Circadian phase resetting by a single short-duration light exposure
}

\author{
Shadab A. Rahman, ${ }^{1,2}$ Melissa A. St. Hilaire, ${ }^{1,2}$ Anne-Marie Chang, ${ }^{1,2}$ Nayantara Santhi, ${ }^{1,2,3}$ \\ Jeanne F. Duffy, ${ }^{1,2}$ Richard E. Kronauer, ${ }^{2,4}$ Charles A. Czeisler, ${ }^{1,2}$ Steven W. Lockley, ${ }^{1,2}$ \\ and Elizabeth B. Klerman ${ }^{1,2}$ \\ 'Division of Sleep and Circadian Disorders, Departments of Medicine and Neurology, Brigham and Women's Hospital, \\ Boston, Massachusetts, USA. ${ }^{2}$ Division of Sleep Medicine, Harvard Medical School, Boston, Massachusetts, USA. ${ }^{3}$ Surrey \\ Sleep Research Centre, Faculty of Health and Medical Sciences, University of Surrey, Guildford, Surrey, United Kingdom. \\ ${ }^{4}$ School of Engineering and Applied Science, Harvard University, Cambridge, Massachusetts, USA.
}

BACKGROUND. In humans, a single light exposure of 12 minutes and multiple-millisecond light exposures can shift the phase of the circadian pacemaker. We investigated the response of the human circadian pacemaker to a single 15-second or 2-minute light pulse administered during the biological night.

METHODS. Twenty-six healthy individuals participated in a 9-day inpatient protocol that included assessment of dim light melatonin onset time (DLMO time) before and after exposure to a single 15 -second $(n=8)$ or 2 -minute $(n=12)$ pulse of bright light $(9,500$ lux; $4,100 \mathrm{~K}$ fluorescent) or control background dim light ( $<3$ lux; $\boldsymbol{n}=6$ ). Phase shifts were calculated as the difference in clock time between the two phase estimates.

RESULTS. Both 15-second and 2-minute exposures induced phase delay shifts [median ( \pm SD)] of $-\mathbf{3 4 . 8} \pm \mathbf{4 7 . 2}$ minutes and $\mathbf{- 4 5 . 4} \pm \mathbf{2 8 . 4}$ minutes, respectively, that were significantly $(P=\mathbf{0 . 0 4})$ greater than the control condition (advance shift: $+22.3 \pm 51.3$ minutes) but were not significantly different from each other. Comparisons with historic data collected under the same conditions confirmed a nonlinear relationship between exposure duration and the magnitude of phase shift.

CONCLUSIONS. Our results underscore the exquisite sensitivity of the human pacemaker to even short-duration single exposures to light. These findings may have real-world implications for circadian disruption induced by exposure to brief light stimuli at night.

TRIAL REGISTRATION. The study was registered as a clinical trial on www.clinicaltrials.org, NCT \#01330992.

Role of funding source: The authors are solely responsible for the collection, analysis, interpretation, and reporting of the data. The content of this manuscript is solely the responsibility of the authors and does not necessarily represent the official views of the funding source (National Center for Research Resources, the National Center for Advancing Translational Science, or the NIH).

Conflict of interest: Possible conflicts of interest for all authors are disclosed in the online supplement (https://doi. org/10.1172/jici.insight.89494DS1).

Submitted: July 11, 2016 Accepted: February 14, 2017 Published: April 6, 2017

\section{Reference information:} JCI Insight. 2017;2(7):e89494. https:// doi.org/10.1172/jci.insight.89494.
FUNDING. Funding for this study was provided by NSBRI HFP02802 and NIH P01-AG09975, R01HL114088 (EBK), RC2-HL101340-0 (EBK, SWL, SAR, REK), K02-HD045459 (EBK), K24-HL105664 (EBK), T32-HL07901 (MSH, SAR), HL094654 (CAC), and AG044416 (JFD). The project described was supported by NIH grant 1UL1 TR001102-01, 8UL1TR000170-05, UL1 RR 025758, Harvard Clinical and Translational Science Center, from the National Center for Advancing Translational Science.

\section{Introduction}

Light is the strongest environmental time cue for resetting the circadian clock in mammals, including humans $(1,2)$. Initial studies examining the phase-resetting effects of light used long-duration (5 hours) and high-intensity ( $~ 9,500$ lux) light (2) because the human circadian system was thought to be less sensitive to light than that of other mammals. While the circadian pacemaker in mice (3), rats (4), hamsters (5), and humans $(6,7)$ is known to respond to a sequence of millisecond flashes (administered over 5 seconds in rats, over 5-60 minutes in mice and hamsters, and over 60 minutes in humans), a single millisecond flash does not reset the mammalian pacemaker at the intensities that have been tested $(3,8)$, suggesting that circadian resetting requires integration of the photic signal over a longer exposure interval.

A nonlinear relationship exists between light exposure (LE) duration and the magnitude of circadian phase resetting responses. A single 12-minute pulse of light can shift the human pacemaker 8 times more 
Enrollment

Screening

Allocation

Intervention

Analysis
Assessed for eligibility $(n=67)$
Excluded $(n=25)$

-Medical screen failure $(n=18)$

-Non-compliance $(n=6)$

- Other $(n=1)$

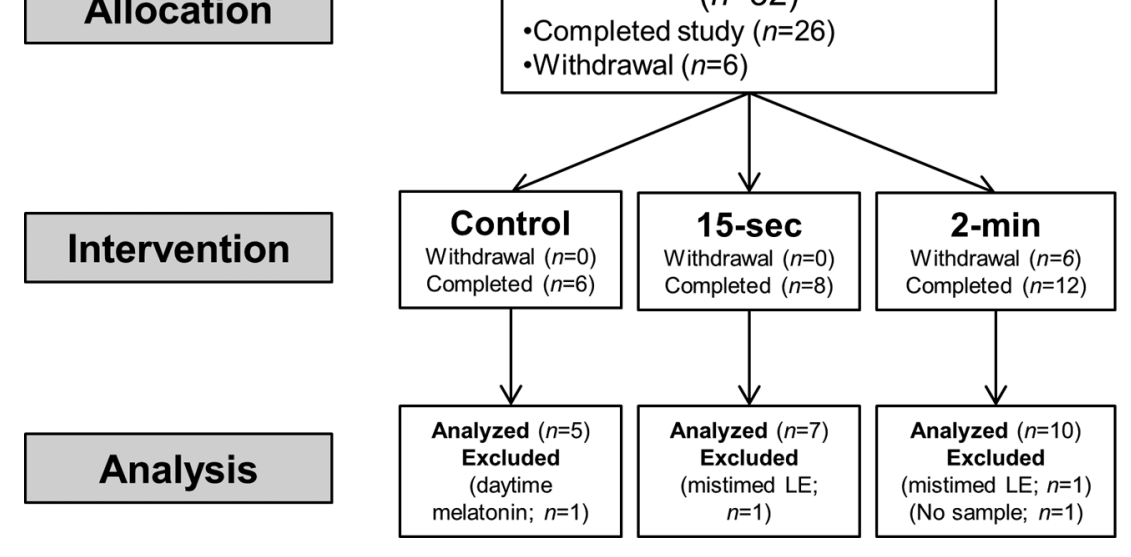

Withdrawal

(time constraints; $n=10$ ) 

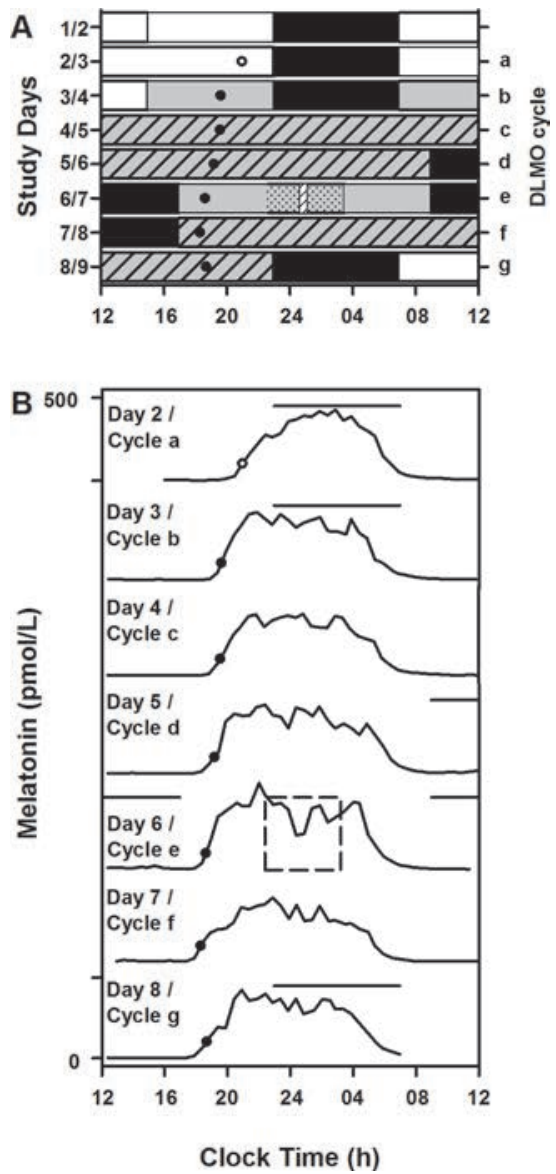

Figure 2. Study protocol and phase shift calculation. All study events were timed according to each individual's schedule, which was maintained for at least three weeks prior to starting the 9-day inpatient part of the study. (A) Example study raster for an individual with 2300-0700 hours habitual sleep. Black bars represent scheduled sleep (time in bed) in darkness, and white bars represent $<200$ lux ambient lighting while awake. Gray bars represent dim light ( $<3$ lux). Gray hashed bars represent constant routine intervals used to assess endogenous circadian phase. The constant posture interval during light exposure is shown as gray dotted bars, and the time of bright light exposure (9,500 lux) is represented by a hashed white bar. Daily dim light melatonin onset (DLMO) times from 1 individual in the control condition is plotted across the days $(\bullet$, under dim light; $\circ$, melatonin crossing the DLMO value under 90 lux room light). (B) Daily melatonin profiles from the same individual plotted serially. The dashed square represents the constant posture interval during which light exposure occurred. All individuals in the control condition received the same dim background ambient lighting and no bright light exposure. Black horizontal lines above the melatonin profiles indicate scheduled sleep. Phase shifts were assessed as the difference in clock time between DLMO on cycles $d$ and $g$.

\pm 47.19 minutes and $-45.44 \pm 28.36$ minutes, respectively) significantly $(P=0.04)$ different from the median phase shift under the dim light control condition $(22.31 \pm 51.36$ minutes $)$ (Figure 3G). The responses to the two LEs were not different from each other, however $(P$ $=0.98$ ). The 4-parameter logistic function fit to the new and historical data (stimulus duration ranging from 15 seconds to 4 hours) had a goodness of fit of $r=0.69$ with estimates ( \pm $\mathrm{SE}$ ) for a (maximum asymptote) $=-3.07 \pm 2.81$ hours, $\mathrm{b}=1.76 \pm 5.62$ hours, $\mathrm{c}$ (minimum asymptote) $=-48.70 \pm 16.01$ minutes, and $\mathrm{d}$ (ratio of minutes of phase shift per minute of bright LE) $=-48.73 \pm 58.01$. This fit estimates the half-maximal phase shift (parameter $b$ ) at a light duration of 1.76 hours, at this light intensity (Figure $3 \mathrm{H}$ ).

The 15-second and 2-minute exposures induced phase delays that represent $\sim 26 \%$ and $\sim 34 \%$ of the resetting observed with a 4-hour exposure, despite representing only $0.10 \%$ and $0.83 \%$, respectively, of the 4 -hour stimulus duration. Thus, compared with the 4-hour pulse, which gave 0.01 minutes phase delay per second of LE, the 15 second pulse was $\sim 230$ times more effective ( 2.32 minutes delay per second of exposure), and the 2 minute pulse was $\sim 38$ times more effective ( 0.38 minutes delay per second of exposure).

\section{Discussion}

Our results show that single bright LEs as short as 15 seconds and 2 minutes induce significant circadian phase shifts in humans. These results also confirm and further refine the nonlinear duration response function to light previously reported (9). To our knowledge, this is the first study to demonstrate a significant phase-resetting response to a single short-duration (15 seconds) light pulse.

The nonlinear relationship between exposure duration and the degree of circadian phase shift has been modeled previously using a 4-parameter logistic function and showed an approximately linear response between 12 minutes and 4 hours (9). Our new results confirm a nonlinear logistic relationship when shorter pulses are added to the function. Our fit estimated the half-maximal phase shift at $\sim 1.76$ hours LE duration, at this light intensity, markedly shorter than the previously reported regression estimate of $\sim 2.7$ hours (9).

The higher-than-expected phase resetting capacity of the short-duration pulses ( $\leq 12 \mathrm{~min})$ suggests that most of the phase resetting in a long--duration pulse likely occurs at the onset of the LE. This supports the Kronauer model (12) of the effects of LE on phase resetting in humans, which predicts that there is a greater activation rate of light-sensitive elements (a measure of resetting effectiveness of light) at the very beginning of a light pulse, defined as the "prompt response" (12). In this model, as activator elements are depleted into a "used" state, the light drive to the pacemaker becomes limited by the rate at which elements are recycled back to a "ready" state, and it is maintained asymptotically marginally above baseline nonactivated levels, defined as the sustained drive (12). The rate of decline from the prompt to the sustained drive follows a nonlinear pattern. Our results in humans are consistent with results from animal studies that showed light-induced phase resetting to be most efficient at the beginning of the LE, with minimal additional phase shift produced by further extension of the light stimulus due to a reduction in photic responsiveness of the circadian pacemaker $(3,4,8,13)$. A recent report documents that the number of light-evoked action potentials per photon from intrinsically photosensitive retinal ganglion cells (ipRGCs) that mediate LE-induced phase shifts declines as the duration of the photic stimulus increases (14). This is likely related to the light adaptation of ipRGCs with sustained photic stimulation (15). These results 

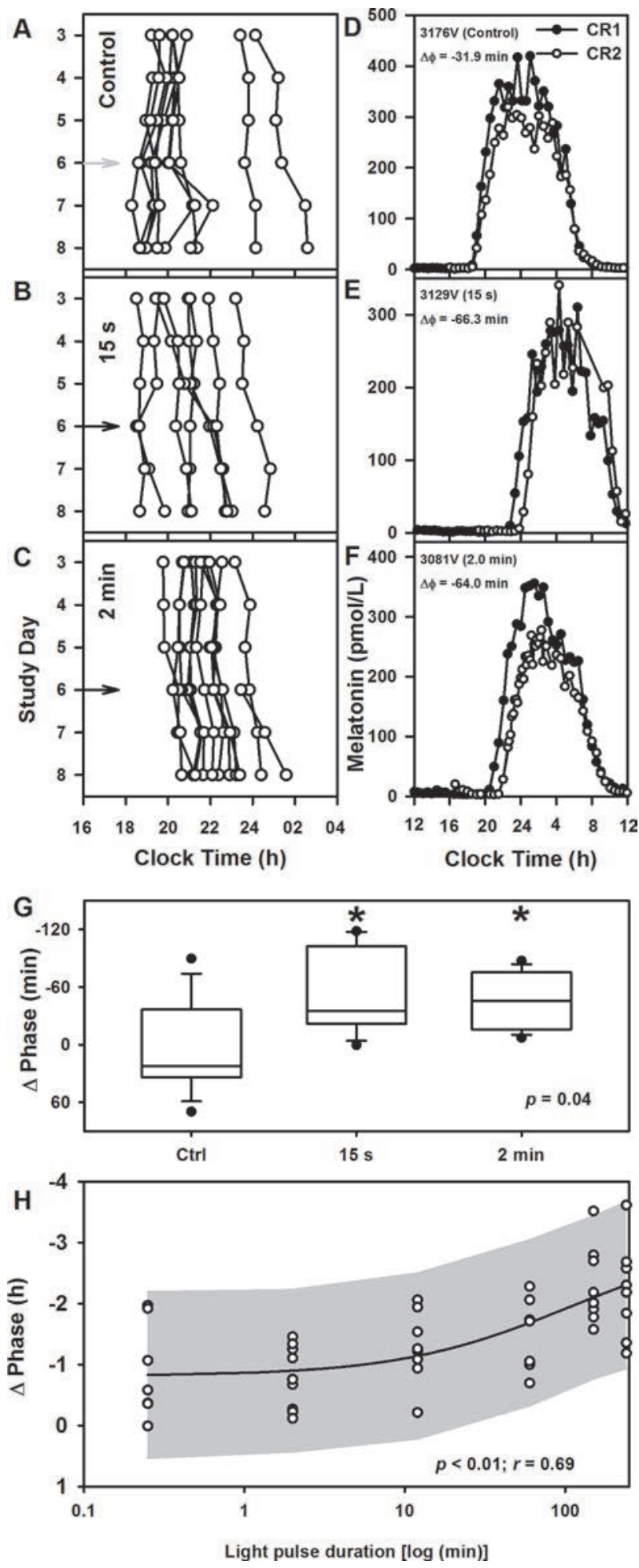

Light pulse duration $[\log (\min )]$
Figure 3. Phase resetting response to short-duration pulses of light and duration response of circadian phase resetting. Daily dim light melatonin onset (DLMOs) times for each individual are shown for (A) dim-light control and (B) 15-second and (C) 2-minute exposure conditions. Light exposure occurred on day 6 (gray arrow, dim light; black arrow, bright light). (D-F) Individual melatonin profiles across $\sim 24$ hours during the $(\bullet)$ first constant routine (CR1) and ( $\circ$ ) second CR (CR2) under each of the three light exposure conditions demonstrate the phase resetting effects of bright-light exposure compared with dim light. (G) Phase shifts of the human circadian system in response to dim-light control $(n=9)$ and to 15 -second $(n=7)$ and 2-minute $(n=10)$ high-intensity night-time light ( 9,500 lux) exposure. The box and whisker plots show the median, 25th and 75th percentile (box limits), the 10th and 90th percentiles (whiskers), and the maximum and minimum points, which are represented as filled circles. ${ }^{*} P<0.05$ by Kruskal-Wallis one-way ANOVA followed by post hoc multiple comparisons using Wilcoxon rank-sum test. All tests were 2-tailed. (H) Phase resetting response (median \pm SD) to 15-second ( $n=7$ ), 2-minute $(n=10), 12$-minute $(n=8), 1$-hour $(n=7), 2.5$-hour $(n=9)$, and 4-hour $(n=8)$ night-time light ( 9,500 lux) pulses fitted with a 4-parameter logistic model. Individual phase shifts are shown as 0 . The $95 \% \mathrm{Cl}$ region of the regression is shown as the shaded area.

provide a cellular explanation for the change in phase resetting efficiency based on the duration of the light pulse as observed in the current study.

Exposure to multiple short pulses $(5,15$, and 45 minutes) delivered over a long-exposure interval (e.g., 6.5 hours) has been shown to induce significant phase shifts in humans $(10,11,16)$. More recently, a train of sixty 2-millisecond pulses administered every minute over $60 \mathrm{~min}$ utes induced $\sim 45$ minutes of phase delay $(6,7)$. Given that the circadian pacemaker can integrate photic signals $(3,8)$, these multiple-exposure studies cannot isolate the phase shift induced by each individual pulse. Mechanistic studies of phase resetting in animals show that the pacemaker can integrate and respond to pulses presented 60 minutes apart (17) and that the majority of a phase-resetting response occurs at the beginning of the light pulse (18-20). Our current results suggest that humans may also be disproportionately sensitive to the first few seconds of a light stimulus (12).

This study had a number of limitations. Firstly, the limited sample size in each LE condition may have precluded detecting differences in phase shifts between the 15-second and 2-minute exposures. Secondly, the magnitude of the shifts in the short-duration pulses is close to the limit of detection for our study techniques. In the current analyses, we assessed phase shifts over three cycles, consistent with previous studies (21-23); however, the endogenous drift over multiple circadian cycles between assessments may influence the phase shift results compared with results calculated across only one or two circadian cycles $(22,24,25)$. Additionally, the studies were performed against a dim light background, which reduces the masking effects of light on the circadian pacemaker but can cause moderate sensitization of the system to subsequent bright LEs $(26,27)$. Although the mean illuminances appeared to differ between the duration conditions with up to a $\sim 45 \%$ difference between the highest mean $(9,040$

lux, 1 hour condition) and lowest mean intensities (6,226 lux, 2 minute condition), it is highly unlikely that this affected the phase resetting responses. Even the lowest mean intensity was more than 10 times higher than the $\sim 500$ lux intensity at which phase resetting responses saturate in studied under conditions that are similar to the current study (28). Moreover, the 1-hour condition had lower phase resetting efficiency than the 15-second and 2-minute conditions, even though the mean intensity of the 1-hour condition was higher than the other two conditions. 
These results have important physiological and clinical implications. Light levels equivalent to ordinary room light ( 100 lux) induce half-maximal phase shifts compared with bright light $(\sim 1,000-10,000$ lux) when the stimuli are 6.5 hours long (28). Results from the current study demonstrate that even short-duration exposures (on the order of seconds) can cause significant phase resetting. Taken together, the results warrant future studies to examine the effects of short-duration LEs at indoor intensities on circadian phase resetting. Such inadvertent phase resetting may be an unrecognized risk factor for insomnia, circadian rhythm sleep disorders, and a variety of adverse health effects associated with circadian rhythm disruption. Alternatively, the effectiveness of these short-light stimuli for resetting circadian rhythms may have potential therapeutic value to improve compliance with treatments for circadian misalignment associated with shift-work, jet-lag, and other circadian rhythm sleep disorders.

\section{Methods}

\section{Participants and prestudy conditions}

Twenty-six young healthy individuals (15 females; mean age \pm SD $=23.6 \pm 3.3$ years; range 19-30 years) completed the protocol (Figure 1) in the Intensive Physiological Monitoring Unit in the Center for Clinical Investigation (CCI) at Brigham and Women's Hospital. The study included three groups: 15-second ( $n=$ 8; 5 female; mean age [ \pm SD], $24.2 \pm 4.3$ years) or 2 -minute $(n=12 ; 6$ female; mean age $[ \pm S D], 23.3 \pm 3.0$ years) LE conditions, and the dim-light control condition ( $n=6 ; 4 \mathrm{~F}$; mean age $[ \pm \mathrm{SD}] 23.8 \pm 2.7$ years). Data from six individuals who voluntarily withdrew prior to study completion were not included in the analyses. All were healthy by physical, psychological, and ophthalmologic exams. For at least three weeks prior to admission, participants maintained a self-selected, consistent 8-hour sleep/rest/dark schedule that was confirmed with calls to a time- and date-stamped voicemail at bedtime and wake time and, for at least 7 days prior to entering the unit, objective monitoring with actigraphy and photometry (Actiwatch-L, Minimitter Inc.). Throughout screening, participants were asked to refrain from the use of any prescription or nonprescription medications, supplements, recreational drugs, caffeine, alcohol, or nicotine. Compliance was verified by urine and blood toxicology during screening and urine toxicology upon entry to the unit.

\section{Study protocol}

Participants were studied for 9 days in an environment free of time cues and were supervised by staff trained not to reveal information about the time of day. The schedule consisted of a 3-day baseline ( 8 hours/16 hours sleep/wake cycle based on average sleep times in the 7 days prior to study entry), an initial 50-hour constant routine circadian phase assessment (CR1, described below), and a 16-hour wake episode during which the experimental LE was presented (LE day). This was followed by an 8-hour scheduled sleep opportunity, a 30-hour CR (CR2), and then a final 8-hour sleep opportunity, after which time they were discharged (Figure 2A). During the CR procedures (29), participants were asked to remain awake while supervised in constant dim light in a semirecumbent posture, with daily nutritional and fluid intake divided into isocaloric hourly portions.

\section{Lighting conditions and experimental LE}

Ambient light conditions. During the first 2.5 baseline days, maximum ambient light during scheduled wake was $\sim 190$ lux $\left(\sim 48 \mu \mathrm{W} / \mathrm{cm}^{2}\right)$ when measured in the horizontal plane at a height of $187 \mathrm{~cm}$ and $\sim 88$ lux $\left(\sim 23 \mu \mathrm{W} / \mathrm{cm}^{2}\right)$ when measured in the vertical plane at a height of $137 \mathrm{~cm}$. Midway through day 3, ambient light was decreased to $<3$ lux maximum ( $1.5 \mathrm{lux}\left[<0.4 \mu \mathrm{W} / \mathrm{cm}^{2}\right.$ to $\left.\sim 0.1 \mu \mathrm{W} / \mathrm{cm}^{2}\right]$ ) when measured in the vertical plane at $137 \mathrm{~cm}$ and maintained at that level for the remainder of the study, except during the brief experimental LEs (see below) and during scheduled sleep opportunities when all lights were turned off (0 lux). The ambient lights were dimmed midway through day 3 in order to assess DLMO time as a phase estimate under entrained conditions $(9,30)$.

Experimental LE conditions. The LE procedure included a constant posture episode during which the bright light pulse was administered. The constant posture was used to negate confounding from ambulatory conditions on research measurements (9). The constant posture began 5.7 hours after wake time on the LE day and lasted for 4.5 hours (to accommodate the longest exposure of 4 hours) (9). The 4.5-hour constant posture interval was approximately centered within the 16-hour wake episode, therefore starting 5.7 hours after wake and ending 5.8 hours before bedtime on that study day. The LE was centered on the 
16-hour wake episode, which occurred 7.9 hours after wake (2.2 hours after the start of the constant posture) for the 15-second and 2-minute pulses, respectively.

During the constant posture, participants remained seated in a specific location of the room and wore clear UVEX glasses (Uvex Winter Optical) to remove UV radiation. During the constant posture interval, when participants remained in dim background light, participants alternated fixed gaze (lasting at least 5 minutes) with an equal interval of "free gaze." During fixed gaze, participants focused on a target on the wall directly in front of them and during free gaze, participants were allowed to look elsewhere as long as they did not close their eyes or shield them from the light. Light illuminance was measured at every change of gaze, with the sensor placed next to the participant's eye and pointed at the target during fixed gaze and in the angle of gaze during free gaze. Starting 10 minutes immediately prior to the light pulse, all participants maintained fixed gaze. During bright light pulses $>2$ minutes, participants continued with their fixed and free gaze pattern, whereas during light pulses $\leq 2$ minutes, participants stared directly above at the ceiling where the lights were located for the duration of the bright light pulse. In the control condition, the same sequence as in the 2-minute exposure was followed. During the 15-second or 2-minute LE within this session, the target corneal illuminance was $>9,500 \operatorname{lux}\left(>8 \times 10^{6} \mu \mathrm{W} / \mathrm{cm}^{2}\right)$; for the control condition, background lighting ( $<3 \mathrm{lux}$ ) was maintained. The mean \pm SD illuminance achieved during the 15 -second pulse was 8,022 \pm 1,387 lux; during the 2-minute pulse, it was 6,226 $\pm 1,546$ lux; and during the control condition, it was $0.5 \pm 0.2$ lux. A research technician was present during the entire LE session to administer the LE, measure and record light readings, and monitor adherence to study procedures.

Technical details of light generation and measurement. Ambient room lighting was generated using ceiling-mounted 4,100 K fluorescent lamps (F96T12/41U/HO/EW, 95W; F32T8/ADV841/A, 32W; F25T8/ TL841, 25W; Philips Lighting) with digital ballasts (Hi-Lume 1\% and Eco-10 ballasts, Lutron Electronics Co. Inc.) transmitted through a UV-stable filter (Lexan 9030 with prismatic lens, GE Plastics). Routine illuminance and irradiance measures were conducted using an IL1400 radiometer/powermeter with an SEL-033/Y/W or SEL-033/F/W detector, respectively (International Light Inc.).

\section{Outcome measures of melatonin phase}

Blood samples were collected every 5-60 minutes throughout the protocol, and the plasma was frozen for later assay. Melatonin concentration was determined by double-antibody radioimmunoassay with the Kennaway G280 antiserum (31) by a laboratory blinded to experimental conditions (Specialty Assay Research Core Laboratory, Brigham and Women's Hospital). The plasma melatonin intra-assay coefficient of variation $(\% \mathrm{CV})$ was $8.5 \%$ at $5.85 \mathrm{pg} / \mathrm{ml}$ and $8.0 \%$ at $25.46 \mathrm{pg} / \mathrm{ml}$, and the interassay $\% \mathrm{CV}$ was $10.8 \%$ at $5.98 \mathrm{pg} / \mathrm{ml}$ and $14.5 \%$ at $23.07 \mathrm{pg} / \mathrm{ml}$.

The DLMO was used as the endogenous circadian phase marker and calculated as the time at which levels of melatonin rose above the $25 \%$ peak-to-trough amplitude threshold of a 3-harmonic waveform fitted to the first melatonin secretory episode during CR1 $(30,32)$. Once the threshold for each individual's DLMO was calculated, it was applied to each daily melatonin onset and described by letter (a-g; see Figure 2B).

\section{Historic data}

In addition to those participants described above, (i) four male participants (21.0 \pm 1.8 years) previously studied in our laboratory using a near-identical protocol under dim light conditions were included in the control condition for the phase shift analysis (22), and (ii) 39 healthy young adults (22.2 \pm 3.6 years; 16 female) previously studied in our laboratory using near-identical procedures in a protocol that examined the phase shifts in response to LEs of 12 minutes, 1 hour, 2.5 hours, and 4 hours were reanalyzed to evaluate a broader duration response of circadian phase resetting (9). The current and historical studies were the same in participant screening criteria and experimental conditions, except for the (i) LE duration and timing and (ii) CR duration. The 4-hour LE was scheduled to begin 0.5 hours after the start of the 4.5 -hour constant posture session (6.2 hours after wake time) and end at the same time as the LE session in this protocol. The 2.5-hour and 1-hour LEs were each scheduled so that the midpoint of the LE occurred at the same time of the midpoint for the 4-hour LE ( 8.2 hours after wake time). The timing of the 12-minute LE occurred slightly earlier, with the midpoint 12 minutes earlier than the other groups. CR1 was a 50-hour wake episode (same as the current study) in the 12-minute condition and a 50.2-hour wake episode in the 1-hour, 2.5-hour, and 4-hour conditions. CR2 was a 30-hour wake episode (same as the current study) in the 12-minute condition and a 29.8-hour wake episode in the 1-hour, 2.5-hour, and 4-hour conditions (9). 
The mean $( \pm$ SD) light intensities for the historic conditions were as follows: 12 minutes was 7,669 $\pm 1,321$ lux, 1 hour was 9,040 \pm 498 lux, 2.5 hours was 8,927 \pm 666 lux, and 4 hours was 8,396 \pm 1,604 lux (9).

\section{Data analysis}

Data are reported as the median $( \pm S D)$ unless otherwise noted. All analyses were performed using SAS 9.3 (SAS Institute Inc.). Data from 22 of the 26 current participants were included in the analysis of melatonin phase shifts. One male participant from the control condition was excluded due to significant daytime melatonin secretion such that a reliable DLMO could not be determined. For two participants ( $n=1$ from each of the two LE groups; both females), post-hoc analysis revealed that the LE was 4.4 hours after DLMO, which was outside the target range (0.1-4.1 hours) for inducing $85 \%$ of the maximal delay shift (22), so they were excluded from analysis. DLMO in the second cycle of CR2 could not be assessed in 1 female participant in the 2-minute exposure group due to missing blood samples resulting from i.v. malfunction. Four participants ( $n=3$ from the 1-hour group and $n=1$ from the 2.5-hour group) from the historical cohort were also excluded from the analysis due to inappropriate timing of the LE as per the target range described above.

Phase shifts were calculated as the difference between the DLMO on the second cycle of CR1 (pre-LE phase) and the DLMO on the second cycle of CR2 (post-LE phase) three cycles later (cycles $d$ and g; Figure 2A and B) (21-23). Median melatonin phase shift data for each LE duration group were fit by a 4-parameter logistic model to characterize the relationship between phase shift and light duration (SAS PROC NLMIXED). The 4-parameter logistic model was previously found to best fit the relationship between phase shift and stimulus duration or intensity $(9,28)$. The equation for the 4-parameter logistic model was as follows: $\mathrm{y}=\left([\mathrm{a}-\mathrm{c}] /\left[1+(\mathrm{x} / \mathrm{b})^{\mathrm{d}}\right]\right)+\mathrm{c}$, where " $\mathrm{a}$ " is the estimated response of the system to a bright light pulse of 0 -second duration, " $\mathrm{b}$ " is the duration at which $50 \%$ of the maximal shift is observed, "c" is the asymptotic maximal responsiveness of the system, and " $\mathrm{d}$ " is a measure of the steepness of the rising portion of the curve.

\section{Statistics}

All statistical analyses were performed using SAS 9.3 (SAS Institute Inc.). Due to the limited sample sizes in each group, phase shift data were analyzed by the nonparametric Kruskal-Wallis one-way ANOVA to assess the differences in median phase shifts between LE conditions. Post hoc multiple comparisons were made using Wilcoxon rank-sum test. All statistical tests were two-tailed with a significance threshold set to $P<0.05$.

\section{Study approval}

The study was reviewed and approved by the Partners Human Research Committee (Boston, Massachusetts, USA), and participants provided written informed consent prior to study.

\section{Author contributions}

JFD, REK, CAC, SWL, and EBK designed research. SAR, MASH, AMC, NS, and JFD performed data collection. SAR, MASH, AMC, NS, JFD, REK, CAC, SWL, and EBK performed data analysis and interpretation. SAR, MASH, AMC, NS, JFD, REK, CAC, SWL, and EBK prepared manuscript.

Address correspondence to: Shadab A. Rahman, Division of Sleep and Circadian Disorders, Departments of Medicine and Neurology, Brigham and Women's Hospital, 221 Longwood Avenue Boston, Massachusetts 02115, USA. Phone: 617.525.8830; E-mail: sarahman@rics.bwh.harvard.edu.

\footnotetext{
1. Klerman EB, Rimmer DW, Dijk DJ, Kronauer RE, Rizzo JF, Czeisler CA. Nonphotic entrainment of the human circadian pacemaker. Am J Physiol. 1998;274(4 Pt 2):R991-R996.

2. Czeisler CA, et al. Bright light induction of strong (type 0) resetting of the human circadian pacemaker. Science. 1989;244(4910):1328-1333.

3. Van Den Pol AN, Cao V, Heller HC. Circadian system of mice integrates brief light stimuli. Am J Physiol. 1998;275(2 Pt 2):R654-R657.

4. Arvanitogiannis A, Amir S. Resetting the rat circadian clock by ultra-short light flashes. Neurosci Lett. 1999;261(3):159-162.

5. Vidal L, Morin LP. Absence of normal photic integration in the circadian visual system: response to millisecond light flashes. J Neurosci. 2007;27(13):3375-3382.

6. Zeitzer JM, Ruby NF, Fisicaro RA, Heller HC. Response of the human circadian system to millisecond flashes of light. PLoS
} 
ONE. 2011;6(7):e22078.

7. Najjar RP, Zeitzer JM. Temporal integration of light flashes by the human circadian system. J Clin Invest. 2016;126(3):938-947.

8. Nelson DE, Takahashi JS. Integration and saturation within the circadian photic entrainment pathway of hamsters. Am J Physiol. 1999;277(5 Pt 2):R1351-R1361.

9. Chang AM, et al. Human responses to bright light of different durations. J Physiol (Lond). 2012;590(13):3103-3112.

10. Gronfier C, Wright KP, Kronauer RE, Jewett ME, Czeisler CA. Efficacy of a single sequence of intermittent bright light pulses for delaying circadian phase in humans. Am J Physiol Endocrinol Metab. 2004;287(1):E174-E181.

11. Rimmer DW, Boivin DB, Shanahan TL, Kronauer RE, Duffy JF, Czeisler CA. Dynamic resetting of the human circadian pacemaker by intermittent bright light. Am J Physiol Regul Integr Comp Physiol. 2000;279(5):R1574-R1579.

12. Kronauer RE, Forger DB, Jewett ME. Quantifying human circadian pacemaker response to brief, extended, and repeated light stimuli over the phototopic range. J Biol Rhythms. 1999;14(6):500-515.

13. Nelson DE, Takahashi JS. Sensitivity and integration in a visual pathway for circadian entrainment in the hamster (Mesocricetus auratus). J Physiol (Lond). 1991;439:115-145.

14. Walch OJ, Zhang LS, Reifler AN, Dolikian ME, Forger DB, Wong KY. Characterizing and modeling the intrinsic light response of rat ganglion-cell photoreceptors. J Neurophysiol. 2015;114(5):2955-2966.

15. Wong KY, Dunn FA, Berson DM. Photoreceptor adaptation in intrinsically photosensitive retinal ganglion cells. Neuron. 2005;48(6):1001-1010.

16. Gronfier C, Wright KP, Kronauer RE, Czeisler CA. Entrainment of the human circadian pacemaker to longer-than-24-h days Proc Natl Acad Sci USA. 2007;104(21):9081-9086.

17. Best JD, Maywood ES, Smith KL, Hastings MH. Rapid resetting of the mammalian circadian clock. J Neurosci. 1999;19(2):828-835

18. Butcher GQ, Lee B, Obrietan K. Temporal regulation of light-induced extracellular signal-regulated kinase activation in the suprachiasmatic nucleus. J Neurophysiol. 2003;90(6):3854-3863.

19. Dziema H, Oatis B, Butcher GQ, Yates R, Hoyt KR, Obrietan K. The ERK/MAP kinase pathway couples light to immediate-early gene expression in the suprachiasmatic nucleus. Eur J Neurosci. 2003;17(8):1617-1627.

20. Butcher GQ, Doner J, Dziema H, Collamore M, Burgoon PW, Obrietan K. The p42/44 mitogen-activated protein kinase pathway couples photic input to circadian clock entrainment. J Biol Chem. 2002;277(33):29519-29525.

21. Khalsa SB, Jewett ME, Cajochen C, Czeisler CA. A phase response curve to single bright light pulses in human subjects. JPhysiol (Lond). 2003;549(Pt 3):945-952.

22. St Hilaire MA, Gooley JJ, Khalsa SB, Kronauer RE, Czeisler CA, Lockley SW. Human phase response curve to a $1 \mathrm{~h}$ pulse of bright white light. J Physiol (Lond). 2012;590(13):3035-3045.

23. Wright KP, Czeisler CA. Absence of circadian phase resetting in response to bright light behind the knees. Science. 2002;297(5581):571

24. Jewett ME, Rimmer DW, Duffy JF, Klerman EB, Kronauer RE, Czeisler CA. Human circadian pacemaker is sensitive to light throughout subjective day without evidence of transients. Am J Physiol. 1997;273(5 Pt 2):R1800-R1809.

25. Duffy JF, Kronauer RE, Czeisler CA. Phase-shifting human circadian rhythms: influence of sleep timing, social contact and light exposure. J Physiol (Lond). 1996;495:(Pt 1):289-297.

26. Smith KA, Schoen MW, Czeisler CA. Adaptation of human pineal melatonin suppression by recent photic history. J Clin Endocrinol Metab. 2004;89(7):3610-3614.

27. Chang AM, Scheer FA, Czeisler CA. The human circadian system adapts to prior photic history. J Physiol (Lond). 2011;589(Pt 5):1095-1102.

28. Zeitzer JM, Dijk DJ, Kronauer R, Brown E, Czeisler C. Sensitivity of the human circadian pacemaker to nocturnal light: melatonin phase resetting and suppression. J Physiol (Lond). 2000;526 Pt 3:695-702.

29. Duffy JF, Dijk DJ. Getting through to circadian oscillators: why use constant routines? J Biol Rhythms. 2002;17(1):4-13.

30. Lockley SW, Brainard GC, Czeisler CA. High sensitivity of the human circadian melatonin rhythm to resetting by short wavelength light. J Clin Endocrinol Metab. 2003;88(9):4502-4505.

31. Vaughan GM. New sensitive serum melatonin radioimmunoassay employing the Kennaway G280 antibody: Syrian hamster morning adrenergic response. J Pineal Res. 1993;15(2):88-103.

32. Klerman EB, Gershengorn HB, Duffy JF, Kronauer RE. Comparisons of the variability of three markers of the human circadian pacemaker. J Biol Rhythms. 2002;17(2):181-193. 